\title{
White Light Scanning Interferometry for Nano Surface Metrology
}

\author{
ByoungChang Kim¹, TaeHyun Kim¹, SunHyuck Kim¹, ChangKyu Kim², HyungSuk Lee ${ }^{2}$ \\ ${ }^{1}$ School of Mechanical Engieering, Kyungnam University \\ ChangWon City, South Korea \\ bckim@uok.ac.kr; xogus9210@naver.com; sunhyuck1234@naver.com \\ ${ }^{2}$ NanoSystems Co \\ Daejeon City, South Korea \\ cqkim@nanosystemz.com; hslee@nanosystemz.com
}

\section{Extended Abstract}

Polymer nanofiber composites for the treatment of hazardous compounds are of considerable scientific and technological interest. In this study, polyamide nanofiber for organic pollutant removal and chemical warfare protection is discussed. The effect of position of functional materials in nanofiber matrix on the photocatalytic activity was studied by comparing the Ag$\mathrm{TiO}_{2}$-decorated nylon nanofiber composite (AT-sur-NF) and $\mathrm{Ag}$ - $\mathrm{TiO}_{2}$-embedded nylon nanofiber composite (AT-in-NF) [1]. We find that AT-sur-NF shows better photocatalytic activity compared to the photocatalytic activity of AT-in-NF. Based on these results, nylon and meta-aramid nanofibers decorated by various functional nanomaterials were fabricated. The electrospun meta-aramid nanofiber composites exhibit poor chemical stability because of the salt molecules remaining between meta-aramid chains [2]. The chemical stability of meta-aramid nanofiber composites were improved by removing salt molecules during washing and additional thermal treatment. The polyamide nanofiber composites were stacked to enhance mechanical properties and resistivity to chemical warfare agents (CWAs). By controlling the stacking of polyamide nanofiber composites, thickness, weight density, and cool/warm feeling are optimized. In addition, the assemblies exhibit enough resistivity to CWAs while still maintain water vapor transmission to allow evaporation of sweat on the skin. Further study on the thermal properties and microstructure of nylon nanofibers reveals that the chains rigidity and thermal stability increase with decreasing diameter of nylon nanofibers.

\section{References}

[1] S.-Y. Ryu, J. W. Chung, S.-Y. Kwak, "Dependence of photocatalytic and antimicrobial activity of electrospun polymeric nanofiber composites on the positioning of $\mathrm{Ag}-\mathrm{TiO}_{2}$ nanoparticles," Composite Science and Technology, vol. 117, pp. 9-17, 2015.

[2] S.-Y. Ryu, M, Park, S.-Y. Kwak, "Effect of salt removal and heat-pressing treatments on mechanical properties of electrospun meta-aramid nanofibres," Int. J. Nanotechnol., vol. 13, no. 6, pp. 426-437, 2016. 